\title{
The Be Phenomenon
}

\section{L.A. Balona \\ South African Astronomical Observatory, PO Box 9, Observatory 7935, Cape Town, South Africa}

\begin{abstract}
An overview of the Be phenomenon is given with particular emphasis on the problem of the mass loss mechanism. We assume that the principal mechanism of mass loss in these stars is magneto-centrifugal acceleration (MCA). Within this framework, we discuss how particular features of Be stars which distinguish them from normal B stars can be understood. The frequency distribution of $\mathrm{Be}$ stars relative to normal B stars in the Galaxy and Magellanic Clouds is discussed. The distribution of equatorial velocities, activity in the visual and X-ray regions and photospheric activity all point towards a magnetic model and seem to be qualitatively consistent with the MCA hypothesis. Periodic variations in light and line profiles offer particularly important clues. In this respect, the recent discovery of co-rotating photospheric clouds in several Be stars strongly suggests gas trapped in closed field lines. We investigate the dynamics of an outburst and suggest that the localized nature of these events imply magnetic flaring and acceleration of mass due to connection and disconnection of field lines. Finally, suggestions for further progress are presented.
\end{abstract}

\section{Introduction}

Be stars are normal dwarfs and giant B stars in which the Balmer lines, particularly $\mathrm{H} \alpha$, show emission or incipient emission. We omit supergiants from the definition, since the process giving rise to Balmer emission in these stars probably has a different origin. Struve_(1931) attributed the emission to an extended envelope where the gas is ionized by the UV radiation from the star. The geometry of the envelope was generally thought to be a thin equatorial disk, but it is only recently that this idea has been shown to be correct. The first imaging of a Be disk ( $\gamma$ Cas) was accomplished by Thom, Granes \& Vakili (1986) using the I2T interferometer. Quirrenbach et al. (1997) has recently published interferometric results for seven Be stars which show that the inclination of the disks are in agreement with what is expected from the projected rotational velocities of the stars.

Our current understanding of a Be star is of a normal B star in which sudden events, or outbursts, eject matter into a thin, dense, low velocity, equatorial disk that gives rise to an IR excess, $\mathrm{H} \alpha$ emission and intrinsic polarization. In addition, there is a radiatively-driven, high-velocity, mainly polar wind over the rest of the star which is responsible for blue-shifted lines of high ionization seen 
in the far UV. The origin of the equatorial disk is the central, unsolved, problem in Be stars. In this paper we will review those aspects of Be stars which shed light on this most important problem, highlighting recent developments. At the same time, we will show how the phenomena unique to $\mathrm{Be}$ stars can be understood, in a qualitative way, by the presence of a magnetic field.

\section{Mass loss mechanisms in stars}

The physical processes which lead to mass ejection in stars have been briefly reviewed by Strafella et al. (1998). They are: radiation pressure, centrifugal force, thermal expansion and hydromagnetic waves. These are the only, presently known, mechanisms which are capable of directly accelerating matter to escape velocities. All other mechanisms act to enable one of these four mechanisms. For example, nonradial pulsation (NRP) cannot, by itself, eject matter from the star since the pulsational velocities are far too small. Most theories invoking NRP use NRP to feed energy into the equatorial surface layers to accelerate them to the critical rotational velocity. It is the centrifugal force mechanism which is ultimately involved.

The only theory for Be star disks which has been worked out in detail is the wind-compressed disk (WCD) theory (Bjorkman \& Cassinelli 1993). Here, the mechanism is radiation pressure: the confluence of hot, radiatively-driven winds from high latitudes as they converge on the equator. In a rapidly rotating star the polar regions are hotter than the equator, so that one can expect a dependence of mass loss on rotation, as observed in Be stars. The main problem with the WCD model is that it does not lead to a disk with sufficient density unless a magnetic field is introduced (Porter 1997). Owocki, Gayley \& Cranmer (1998) have shown that the introduction of gravity darkening leads to a wind which is directed along the poles, rather than the equator, as demanded by the WCD theory. The WCD theory, as it stands, clearly needs to be revised in the light of these problems. Even if it can be successfully revised, it is at best a partial solution, since it does not take into account the episodic nature of the disk formation.

The radiation pressure mechanism, dominant in O-type stars, drops rapidly in efficiency towards the late B-type stars. The centrifugal acceleration is already stronger than radiative acceleration at B0 (Strafella et al. 1998). However, the equatorial velocities of Be stars are well below the critical rotational velocity, so we need some mechanism which acts to extend the radius of rigid rotation. A natural candidate to fulfill this role would be a sufficiently strong large-scale magnetic field interacting with an ambient ionized gas. The effective temperatures of B stars are sufficient to ionize hydrogen up to a considerable distance from the star. Material ejected from the star will therefore remain almost fully ionized and conducting. This is important as the radius of rigid rotation can only be maintained in a conducting medium. The radius of ionization drops towards the late B-type stars, so that a different mechanism needs to be invoked for the Herbig Ae stars. Indeed, there is no convincing observational evidence for equatorial outflow disks in Herbig Ae stars.

According to this picture, the O-type stars lose mass by radiative acceleration, the B-type stars by the process discussed above which we call magneto- 


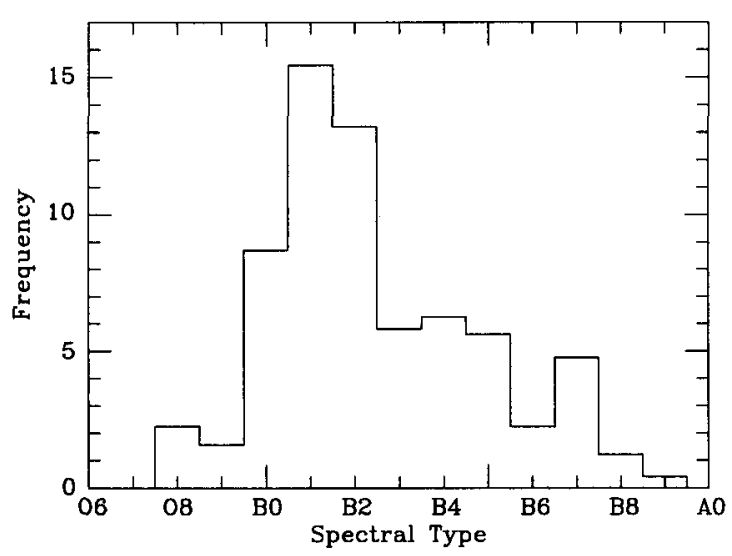

Figure 1. The percentage of classical Be stars among normal B stars in the Galaxy.

centrifugal acceleration (MCA), while the Herbig Ae stars require a third mechanism, which Strafella et al. (1998) suggest may be hydromagnetic waves. We will interpret the Be phenomenon in this framework, more fully discussed in Balona \& Böhm (2000).

\section{The proportion of Be stars relative to normal $\mathrm{B}$ stars}

There is a strong dependence of the relative proportions of Be stars to normal B stars as a function of spectral type. Zorec \& Briot (1997) has discussed the frequency distribution of Be stars taking into account errors in spectral type determination, etc. They find that the distribution is independent of spectral class, implying that the stars are not confined to a particular age. We have used the General Catalogue of MK Classifications (Buscombe \& Foster 1995) to calculate the statistics. The result, from a total of 4035 O7-B9 stars, of which 282 are Be stars, is shown in Fig 1 .

In terms of the MCA mechanism, the decrease in the relative numbers of $\mathrm{Be}$ stars earlier than $\mathrm{B} 1$ is a result of the increasing effectiveness of the radiativelydriven wind which tends to remove the Be disk. Because the the ionization radius decreases for cooler stars, the cooler stars need to rotate closer to their critical rotational velocity if the co-rotation radius is to fall within the ionization radius. Since there are fewer stars rotating at such high velocities, the relative numbers of Be stars can be expected to decrease with effective temperature, as shown in Fig. 1.

Fig. 2 shows the mean equatorial velocities for B-type stars and for Be stars as a function of spectral type. The data are from Jaschek \& Egret (1982) and Bernacca \& Perinotto (1973). The mean equatorial velocity has been determined on the assumption that the axes of rotation are randomly orientated. Also shown is the approximate critical velocity. We note, as expected, that the late-type 


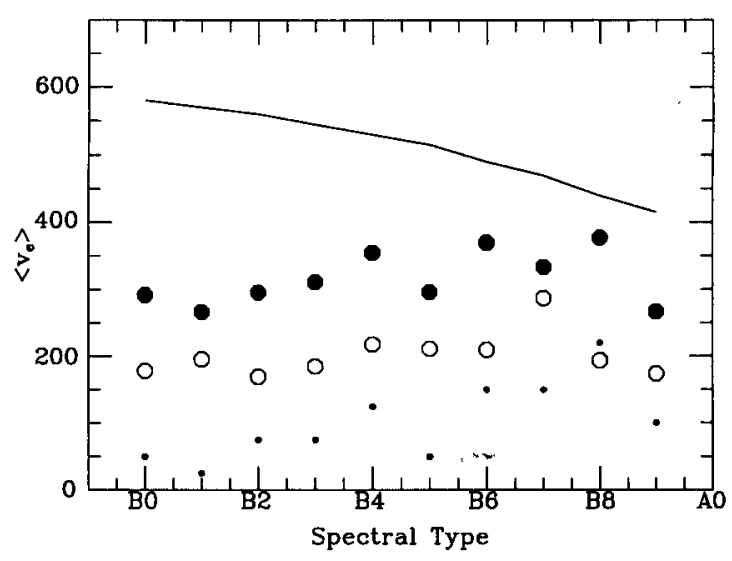

Figure 2. The mean equatorial rotational velocities for B-type stars (open circles) and for Be stars (filled circles) as a function of spectral type. The curve is the approximate locus of the critical rotational velocity. The small filled circles are the approximate minimum observed rotational velocities.

Be stars are indeed rotating much closer to the critical velocity than the earlier types. From these data, Balona \& Böhm (2000) show that the gas in the vicinity of the star is rigidly rotating up to a distance of just over one stellar radius at $\mathrm{B} 9$ to about 1.5 stellar radii at $\mathrm{B} 0$ as measured from the centre of the star.

If the radius of ionization is less than the radius required to attain Keplerian orbital velocity, mass loss cannot occur. For the MCA mechanism to operate, therefore, a star of a given spectral type must be rotating faster than a certain threshold velocity which will be larger for cool stars than for hot stars. In Fig. 2 we have attempted to show the lower limit of equatorial velocities as a function of spectral type. There is indeed a trend in the direction expected for the MCA mechanism. We note that Grady et al. (1987) have concluded, from far UV spectroscopy, that a rotational threshold of about $v \sin i=150$ to $200 \mathrm{~km} \mathrm{~s}^{-1}$ is required for a star to show Be properties. While this limit needs to be revised in the light of Be stars such as $\beta$ Cep $\left(v \sin i=28 \mathrm{~km} \mathrm{~s}^{-1}\right)$, it does support the general existence of a threshold in rotational velocities.

The proportion of Be stars in the Galaxy varies quite significantly from cluster to cluster. In the LMC and SMC the proportion of Be stars in some clusters is particularly large, reaching 50 percent. This has led Maeder, Grebel \& Mermilliod (1999) to conclude that there is a correlation between metal abundance and the proportion of Be stars. Recent work suggests that the high relative abundance in Be stars in the Magellanic Clouds is confined to specific clusters, just as it is in the Galaxy. Keller, Wood \& Bessell (1999) find that the fraction of Be stars in the Magellanic Clouds varies from 0.10 to 0.34, with an average of 0.17 , close to the value for Be stars in the Galaxy. The maximum value also peaks at about B1. Keller, Bessell \& da Costa (2000) suggest that the large 
numbers of Be stars in certain clusters is due to an evolutionary effect. Fabregat \& Torrejón (2000) have come to the same conclusion from observations of the abundances of Be stars in Galactic open clusters.

These important recent results need to be confirmed. If the Be stars are indeed confined to a particular evolutionary stage, this may solve the problem of the high numbers of Be stars in certain Magellanic Cloud clusters, but will also have a very strong bearing on the nature of the mass loss mechanism.

\section{Activity in the visual, UV and X-ray bands}

One of the main characteristics of Be stars is that emission line variations generally occur suddenly and unpredictably, i.e.the mass loss events are episodic in nature. In at least one star ( $\mu$ Cen), however, these events appear to be predictable (Rivinius et al., 1998b). The episodic nature fits in quite well with the presence of a magnetic field. It is thought that disconnection and reconnection of magnetic field lines are responsible for flares in the Sun (Haisch, Strong \& Rodonò 1991) causing ablation and acceleration of material to high velocities. If similar physics occurs in Be stars, the material ejected from flares may be further accelerated by the MCA mechanism to feed the circumstellar disk.

All O-type and early B-type stars are soft X-ray emitters (Berghöffer et al., 1997). In the $O$ stars, $X$-ray luminosity is proportional to bolometric luminosity $\left(L_{X} / L_{\mathrm{Bol}} \approx 10^{-7}\right)$, but B star X-ray luminosities show a lot of scatter. It appears that there is a fundamental difference in the X-ray properties of late B-type stars compared to the earlier spectral types, in that the X-ray flux in late B stars is more variable (Berghöffer et al., 1997). A survey of 27 near main sequence $B$ stars by Cohen, Cassinelli \& MacFarlane (1997) shows that the X-ray luminosity drops sharply for stars later than $\mathrm{B} 2\left(L_{X} / L_{\mathrm{Bol}} \approx 10^{-8.5}\right)$, but even so, about 75 percent of the sample have detectable X-rays. The X-ray luminosities of these mid- and late-B stars pose serious problems for an explanation in terms of radiation-driven wind instabilities, since radiatively driven winds should be very weak or absent in these cool stars. All seven Be stars in their list are detected, which is too small a sample to determine whether Be stars have larger X-ray luminosities than normal $B$ stars of the same spectral type. Unlike the visual region, differences between $\mathrm{Be}$ stars and normal $\mathrm{B}$ stars are very difficult to distinguish in the $\mathrm{X}$-ray and far-UV regions.

The distinct differences between O-type, early B-type and late B-type stars in the X-ray band argues in favour of three different physical processes: radiation pressure, the MCA mechanism and hydromagnetic waves respectively. It is possible that the X-ray flux in B stars is mostly of a thermal nature due to magnetic heating (chromospheric activity) rather than wind instabilities, implying that all $B$ stars have magnetic fields. What makes a Be star may be the combination of rapid rotation and magnetic field of sufficient strength to enforce co-rotation up to the Keplerian radius.

X-ray "flares" have been detected in some Be stars such as $\gamma$ Cas. This star is well known to undergo rapid, chaotic X-ray fluctuations. There is evidence that the source of the X-ray flaring is on, or close, to the photosphere rather than from a compact companion (Smith, Robinson \& Corbet 1998a, Smith, Robinson \& Hatzes 1998b, Smith \& Robinson 1999). It is clearly important to 
determine the source of the flaring. While the existing suggests a photospheric origin, more work needs to be done to confirm this. If a clear photospheric origin can be demonstrated, it would be difficult to understand X-ray flaring unless a magnetic process is involved.

\subsection{Periodic variability}

Periodic light variations consistent with the rotational period are present in about half of the early Be stars. There appears to be a drop in the frequency of these variables (the $\lambda$ Eri stars) for mid- and late-B stars. From the surveys by Cuypers, Balona \& Marang (1989) and Balona, Cuypers \& Marang (1992) we find that 14 out of $24 \mathrm{Be}$ stars (58 percent) in the spectral range B0-B3 are $\lambda$ Eri variables. For B4-B6 emission-line stars the percentage rises to 88 percent ( 7 out of $8 \mathrm{Be}$ stars), while in the range B7-B6 only 2 out of 16 stars are $\lambda$ Eri variables (12.5 percent). Apart from the small sample of mid-B stars, this is clear evidence that the incidence of periodicity drops quite sharply for the late Be stars. A spectroscopic survey by Baade (1989) failed to find any sign of short-period line profile variations in a sample of 22 late B-type stars, confirming this conclusion.

About half of the $\lambda$ Eri stars show double-wave light curves. A star may change from a single-wave to a double-wave or vice-versa on a timescale of a few months (Balona, Sterken \& Manfroid 1991). The double-wave light curve, so characteristic of these stars, can be understood if the star has a dipole field. Gas trapped in closed loops will cause co-rotating circumstellar clouds (see below). The amount of gas in the two hemispheres may be responsible for the great variability in the shapes of the light curves.

Photometric studies require only small telescopes and, as a result, many Be stars have been observed over a long time base. These studies have nearly always reported single periods. By contrast, most of the spectroscopic studies have reported multiple periods. Part of the reason may perhaps be found in the tendency to assume the NRP mechanism and interpret all variations as a combination of multiperiodic sinusoid variations. This is implicitly done, for example, in the use of standard period finding algorithms such as CLEAN. Naturally, singly-periodic solutions of a more complex nature are excluded in this way. Another reason for the reported multiple periods in spectroscopic work is the poor sampling of data owing to the limited availability of observing time on medium-sized telescopes. Finally, the physical quantity that is measured is not immediately clear in spectroscopic work. For example, measurement of the position of the peak in a spectral line or the intensity variation across the line profile presupposes a certain model which may not be correct. A good example of these problems is illustrated by the case of $\eta$ Cen (Balona 1999). In this star there is no doubt that only one period is present (the period of rotation) which also has a very large photometric amplitude. Yet the spectroscopic variation is of a complex nature and could be mistaken for multiple periods if analysed in the usual way. The multiperiodicity reported in $\mu$ Cen is almost certainly of this kind as well (Balona et al., 2000).

The dominant period in the line profile variations is usually the same as the photometric period. However, in at least one case this is not true. In $28 \mathrm{CMa}$, for example, the photometric period of $1.48 \mathrm{~d}$ is only found in lines formed in 
the upper atmosphere or inner circumstellar material (Štefl et al., 1998). This period is not always present in the line profiles and only marginally present in the photometry (Štefl, Aerts \& Balona 1999). The dominant 1.37-d period, which is always present in the line profiles, is found in the photospheric lines and is also marginally present in the photometry. The transient period is clearly confined to the upper atmosphere or inner disk and clearly has a different origin from the dominant 1.37-d period.

Since many Be stars are located in the $\beta$ Cep, SPB or $\zeta$ Oph instability strips, one can expect some stars to pulsate. $\beta$ Cep is an obvious example and $\zeta$ Oph, also a Be star, is almost certainly a multimode pulsator of high spherical harmonic degree (Balona \& Kambe 1999). Another example is $27 \mathrm{CMa}$ (Balona $\&$ Rozowsky 1991) which abruptly became a $\beta$ Cep star a few years ago. Note that $\beta$ Cep and $\zeta$ Oph stars are predicted to be unstable due to the opacity mechanism only for short period p-modes, while the SPB stars appear to be stabilized by rapid rotation. Therefore neither of these three types of pulsating stars can account for the long period g-modes which are required for the periodic Be stars. Balona \& Dziembowski (1999) have examined models of early-type B stars for pulsational instability with periods corresponding to those seen in Be stars, but all the models were stable at these periods. In terms of the NRP model, pulsational driving in Be stars cannot be understood as an ionization mechanism.

\section{Co-rotating photospheric clouds}

Recently, Balona (1999) found that the line profile variations in $\eta$ Cen behaved in a way quite differently from NRP. Standard periodogram analysis of the radial velocity, centroid and intensities across the line profile give very uncertain results which could be interpreted as multiperiodicity, as already mentioned. Yet this is at variance with the light curve which shows just a single period of large amplitude ( $0.05 \mathrm{mag}$ in $y, 0.10 \mathrm{mag}$ in $u$ ). In terms of NRP, it is difficult to understand why the line profiles should not be dominated by the very large amplitude photometric period.

By removing the mean profile from the spectral lines and phasing the residual "difference" profiles according to period, it was found that a coherent pattern was obtained only at one period - the 0.64-d photometric period. The reason why the period is difficult to detect using conventional period analyses is due to the complex nature of the variation which is far from sinusoidal. The pattern formed by stacking difference spectra with the photometric period is quite different from NRP. There is residual emission on the red wing for half the cycle and on the blue wing for the other half of the rotational cycle. Residual emission also appears a little distance bluewards of line centre and disappears at the same distance redwards of line centre. Such behaviour is inconsistent with NRP, but can be understood in terms of two co-rotating clouds, one at the equator and the other near the poles. Emission arises when a cloud is on the limb of the star.

Further evidence for co-rotating clouds comes from Balona \& Kaye (1999) who re-analyzed line profile data of $\zeta$ Tau by Kaye \& Gies (1997). Equatorial and polar clouds also seem to be present in this star. A crude model is able to mimic the observations quite well. Finally, Smith et al. (1998a, b) and Smith 
\& Robinson (1999) present evidence for the passage of cool, co-rotating clouds in $\gamma$ Cas, probably associated with X-ray active centres. Co-rotating clouds are also suggested as an explanation for the line profile variations in $\mu$ Cen (Balona et al., 2000).

The evidence for co-rotating clouds is very strong in $\eta$ Cen and $\zeta$ Tau, where NRP cannot fit the pattern of variability. Much work needs to be done to resolve the important question of whether co-rotating clouds can explain the light and profile variations in other stars as well. It should be noted, however, that a very large number of spectra in a comparatively short period of time is required for this work. One can always fit a multiperiodic model, but multiperiodicity should not be accepted without checking other possibilities and without ensuring that the same periods are present in independent data sets.

If the periodic light and line profile variations can be modeled by co-rotating clouds in most Be stars, then we have extremely strong evidence for a magnetic field since no other mechanism can enforce co-rotation. This, in turn, will strongly support the MCA mechanism.

\section{Anatomy of an outburst}

Light curves of outbursts are common. Hubert \& Floquet (1998) have examined the HIPPARCOS data for short-lived (days to weeks) and long-lived (several months) outbursts. They find that short-lived outbursts are generally confined to the early-type Be stars. The inverse behaviour, temporary fading events, seem to be most common among the most rapidly rotating Be stars. It should be noted that the MACHO project has detected many Be stars in the LMC which show a rather smooth long-term light variation consisting of a rapid rise in light followed by a gradual fading to the original light level (Cook et al., 1995). There appears to be no Galactic counterpart for these "bumpers". In general, long-lived outburst light curves in Galactic Be stars are far less regular.

High-resolution spectroscopic observations which capture the initial few hours of an outburst in detail are rare. These are essential if we wish to investigate how an outburst occurs. Perhaps the most important question is whether an outburst involves a global event or whether it is localized and, if so, where. A strong clue that outbursts are local comes from intensive photometric observations of $\kappa \mathrm{CMa}$ (Balona 1990). A mini-outburst was seen in which the rising branch of the light curve consists of an oscillating light wave with a period of several hours, consistent with the rotational period of the star. The implication is that the outburst occurred in a localized region of the photosphere which comes in and out of view as the star rotates.

Rivinius et al. (1998a) have given a detailed account of spectroscopic changes in the $\mathrm{H} \alpha$ line during outbursts in $\mu$ Cen. The outburst is preceded by a sudden decrease in emission peak height and broad emission wings. The outburst itself is defined by a rapid increase in emission-line strength, rapid cyclic $\mathrm{V} / \mathrm{R}$ variability, high-velocity absorptions and a rapid increase in emission peak separation. A gradual relaxation phase then follows.

A higher time-resolved picture of a mini-outburst in $\eta$ Cen is presented by Balona (1999). The event occurred on the night of 1998, 2/3 April when a sudden, substantial decrease of $\mathrm{EW}$ of the $\mathrm{H} \gamma$ (and, to a lesser extent of $\mathrm{H} \beta$ ) 

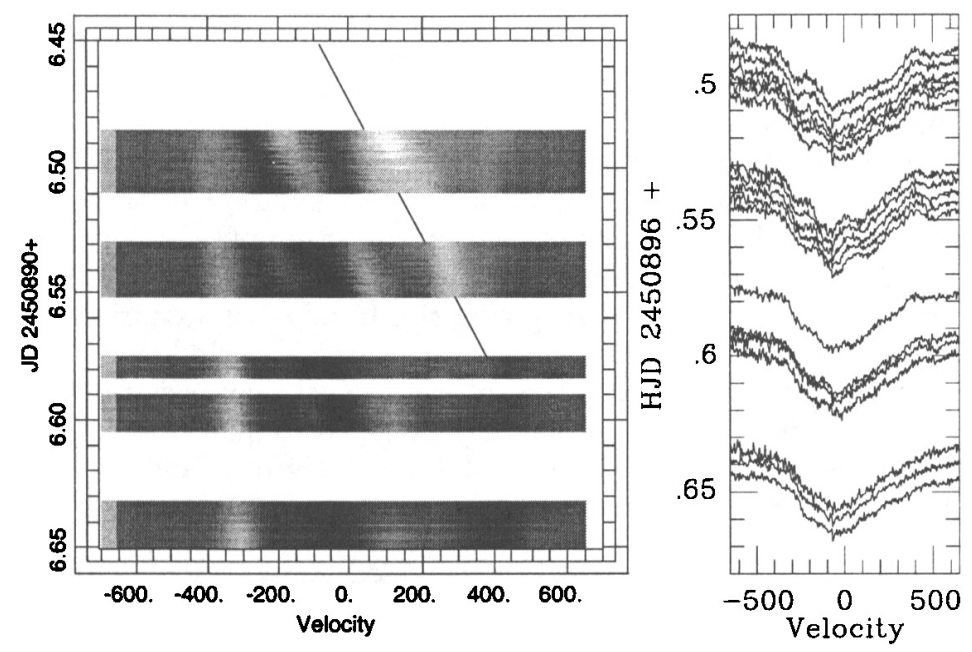

Figure 3. Left: greyscale image of difference profiles of the He I 4388 line in $\eta$ Cen for the night of JD 2450896 showing a flare event in the marked emission lane. Right: corresponding line profiles showing transient moving subfeatures.

occurred. This was accompanied by a sudden increase in the radial velocity amplitude of the hydrogen lines, at that time in pure absorption. Greyscale "difference" spectra during the night in question show that the outburst can be traced to very localized enhanced emission in one of the clouds (Fig. 3). On other nights, the same features were observed, but at much lower intensity. In Fig. 3 several emission lanes are seen with approximately equal spacing, giving rise to strong moving subfeatures which could easily be mistaken for high-degree NRP (Fig. 3). Yet on other nights these subfeatures were very much weaker or absent. The flaring event in this star strongly suggests that a magnetic field is somehow involved.

\section{Conclusions}

Until we discover the cause of the mass loss which gives rise to the equatorial disk in Be stars, our understanding of these stars will be severely compromised. The solution probably revolves around the mechanism which gives rise to the periodic variations. It can no longer be assumed, as was the case until recently, that the role of magnetism can be ignored. The work on $\beta$ Cep by Henrichs et al. (2000) has shown that a magnetic field exists in this Be star. The discovery of periodic X-ray emission from $\theta^{1}$ Ori $\mathrm{C}$ (Gagné et al., 1997) strongly indicates the presence of a magnetic field in this O7V star, a conclusion supported by Babel \& Montmerle (1997). The periodic variations in the Be (also putatively Bp-type) star $\sigma$ Ori $\mathrm{E}$ has long been known to be due to material trapped in 
a dipolar magnetic field. Apart from this, there is now ample indirect evidence for magnetic fields in normal Be stars, as we have shown.

The question therefore arises as to whether one can understand most of the observational properties of Be stars in terms of a magnetic field, or whether a combination of NRP and magnetic field is required. In this paper we suggest that all the observations can be qualitatively understood in terms of a weak dipolar field. Magnetic disconnection and reconnection of field lines gives rise to flaring and ejects material. The material, being highly ionized, is either trapped in closed field lines around the star, giving rise to lobes of obscuring co-rotating gas clouds (periodic Be stars), or else rises along an open field line in another part of the star. At a certain radius, the co-rotating velocity exceeds the local orbital velocity, resulting in a low outflow velocity and a cool equatorial disk. Magnetic centrifugal acceleration seems to account for the Be disk and the presence of a magnetic field can also account for the phenomena previously attributed to NRP.

Future progress is likely to depend on intensive time coverage with high resolution spectroscopy and photometry on a few selected targets. The discovery of co-rotating gas clouds needs to be followed up and extended to more objects. The idea that Be stars are confined to a particular evolutionary stage is extremely important but needs more confirmation. In particular, one has to make sure that the conclusion is independent of the fact that the number of $\mathrm{Be}$ stars happens to peak at B1. We need a theory of mass loss in the presence of a magnetic field in a rotating star. Finally, we need to examine more deeply ways in which magnetic fields can be generated in early-type stars.

\section{References}

Baade D., 1989, A\&A, 222, 200

Babel J., Montmerle T., 1997, ApJ, 485, L29

Balona L.A., 1990, MNRAS, 245, 92

Balona L.A., 1999, MNRAS, 306, 407

Balona L.A., Rozowsky J., 1991, MNRAS, 251, 66p

Balona L.A., Dziembowski W.A., 1999, MNRAS, 309, 221

Balona L.A., Kambe E., 1999, MNRAS, in 308, 1117

Balona L.A., Kaye A.B., 1999, ApJ, 521, 407

Balona L.A., Böhm T., 2000, MNRAS, submitted

Balona L.A., James D., Lawson W.A., Shobbrook R.R., 1999, these proceedings, p. 220

Balona L.A., Cuypers J., Marang F., 1992, A\&AS, 92, 533

Balona L.A., Sterken C., Manfroid J., 1991, MNRAS, 252, 93

Berghöfer T.W., Schmitt J.H.M.M., Danner R., Cassinelli J.P., 1997, A\&A, 322, 167

Bernacca P.L., Perinotto M., 1973, Contrib. Oss. Asiago No 294

Bjorkman J.E., Cassinelli J.P., 1993, ApJ, 409, 429 
Buscombe W., Foster B.E., 1995, General Catalogue of MK Classifications, Northwestern University, Evanston, Illinois

Cohen D.H., Cassinelli J.P., MacFarlane J.J., 1997, ApJ, 487, 867

Cook K.H., et al., 1995, IAU Colloq. 155: Astrophysical Applications of Stellar Pulsations, ed R.S. Stobie, P.A. Whitelock, ASP Conference Series, 83, p.221

Cuypers J., Balona L.A., Marang F., 1989, A\&AS, 81, 151

Fabregat J., Torrejón J.M., 2000, A\&A, 357, 451

Gagné M., Caillault J-P., Stauffer J.R., Linsky J.L., 1997, ApJ, 478, L87

Grady C.A., Bjorkman K.S., Snow T.P., Sonneborn G., Shore S.N., Barker P.K., 1989, ApJ, 339, 403

Haisch B., Strong K.T., Rodonò M., 1991, Ann. Rev. Astron. Astrophys., 29, 275

Henrichs H.F., de Jong J.A., Donati J.F. et al., 2000, these proceedings, p.324

Hubert A.M., Floquet M., 1998, A\&A, 335, 565

Jaschek M., Egret D., 1982, IAU Symp 98, 261

Keller S.C., Wood P.R., Bessell M.S., 1999, A\&AS, 134, 489

Keller S.C., Bessell M.S., Da Costa G.S., 2000, these proceedings, p.75

Kaye A.B., Gies D.R. 1997, ApJ, 482, 1028

Maeder G., Grebal E.K., Mermilliod J-C., 1999, A\&A, 346, 459

Owocki S.P., Gayley K.G., Cranmer S.R., 1998, Boulder-Munich II: Properties of Hot, Luminous Stars, ed I.D. Howarth, ASP Conference Series, 131, p.237

Porter J.M., 1997, A\&A, 324, 597

Quirrenbach, A., Bjorkman, K.S., Bjorkman, J.E., Hummel, C.A., Buscher, D.F., Armstrong, J.T., Mozurkewich, D., Elias, N.M.II., \& Babler, B.L. 1997, ApJ, 479, 477

Rivinius Th., Baade D., Štefl S., Stahl O., Wolf B., Kaufer A., 1998a, A\&A, 333,125

Rivinius Th., Baade D., Štefl S., Stahl O., Wolf B., Kaufer A., 1998b, A\&A, 336,177

Smith M.A., Robinson R.D., Corbet R.H.D., 1998a, ApJ, 503, 877

Smith M.A., Robinson R.D., Hatzes A.P., 1998b, ApJ, 507, 945

Smith M.A., Robinson R.D., 1999, ApJ, 517, 866

Štefl S., Baade D., Rivinius Th., Stahl O., Wolf B., Kaufer A., 1998, A Halfcentury of Stellar Pulsation Interpretations, ed. P.A. Bradely, J.A. Guzik, ASP Conference Series, 135, p.348

Štefi S., Aerts C., Balona L.A., 1999, MNRAS, 305, 505

Strafella F., Pezzuto S., Corciulo G.G., Bianchini A., Vittone A.A., 1998, ApJ, 505,299

Struve O., 1931, ApJ, 73, 94

Thom C., Granes P., Vakili F., 1986, A\&A, 165, L13

Zorec J., Briot D., 1997, A\&A, 318, 443 


\section{Discussion}

Haisch: What is the typical mass loss rate of Be star?

Balona: About $10^{-8} \mathrm{M}_{\odot} \mathrm{yr}^{-1}$.

Zickgraf: In working out the statistics of the relative numbers of Be stars, how is a Be star defined? Is the statistics based on an homogeneous data set? This may be important if there is a dependence of the numbers of Be stars on metallicity.

Balona: Once a star has been observed to show Balmer emission, it remains classified as a Be star, even if the emission disappears. The data set in the General Catalogue of MK Classifications is not biased in any particular way and simply lists the MK types of stars that have bèen classified. All are Galactic Population I stars of approximate solar metallicity.

Dudorov: What can one say about acoustic processes in Be stars?

Balona: It is generally assumed that convection is not present in the atmospheres of Be stars and that the formation of a chromosphere/corona by acoustic and hydromagenetic heating does not take place. If we are forced to accept the presence of magnetic fields in some B stars, this implies turbulent motions at the photosphere (or not too far below the photosphere) in which they can be generated by the dynamo mechanism. Acoustic heating, and a chromosphere/corona, may then be possible. All this, of course, is speculative at the moment, but is being forced on us by the sheer weight of circumstantial evidence showing that magnetic fields must play a role.

Zorec: Two comments: (i) in Zorec \& Briot (1997) we found, by correcting the spectral types of Be stars for rotation, that the relative numbers of Be stars reaches a maximum at B1. The distribution is the same for all luminosity classes, $\mathrm{V}$ to III. This implies that the Be phenomenon is independent of luminosity class and that it is not, therefore, confined to a particular evolutionary phase. (ii) Hubert \& Floquet (1998) have shown from HIPPARCOS light curves that the bumper phenomenon is quite frequent among Be stars in our Galaxy.

Balona: (i) Yes, it is possible that the sharply-peaked distribution of Be stars may mimic an evolutionary effect. This needs to be investigated. (ii) Although Galactic Be stars do have outbursts, their light curves seem to me to be more irregular and of a different nature from the smooth bumper light curves. Normal emission outbursts are also seen in the MACHO database.

Baade: The picture we get of Be stars from open clusters is confusing. It has recently been suggested that the Be phenomenon is linked to a particular stage of evolution. Why would the occurrence of co-rotating clouds preferentially develop at a specific age?

Balona: If it turns out that $\mathrm{Be}$ stars are confined to a particular evolutionary stage, and if we accept that mass loss is due to MCA, then the implication is that magnetic fields are generated only at that evolutionary stage. This may not be a problem, but it is too early to speculate on possible causes since at this time we do not know why magnetic fields are generated in these stars. 